\title{
DESIGN AND OPTIMIZATION OF ARM STRUCTURE FOR MOBILE MEDICAL SERVICE ROBOT BASED ON MULTI - TARGET PARTICLE SWARM OPTIMIZATION
}

\author{
Zi-long Deng ${ }^{1}$, Fang Cheng ${ }^{2}$, Man Zhang ${ }^{3}$ \\ 1,2Anqing vocational and technical college, Anqing, China, \\ Anqing Normal University, Anqing, China \\ ${ }^{3}$ Chongqing technology and business institute, Chongqing, China \\ Corresponding author: Zi-long DENG \\ E-mail: dengzlzl@163.com
}

\begin{abstract}
In order to apply the robot to medical institution, a mobile medical service robot arm is designed. Firstly, according to the design requirements and the ergonomic theory, the various configurations of the mechanical arm are analysed. The optimal configuration of the mechanical arm is obtained by using the global relative manipulability value as the index. Secondly, according to the application and function of mobile service robot, the maximum working space distance index, static stiffness performance index and global velocity fluctuation performance index of arm performance are proposed. On this basis, the mathematical function model of robot arm size optimization is established. Finally, according to the characteristics of the multi-objective and multi-constraint of the optimization function model, the multi-objective particle swarm optimization algorithm based on the Pareto dominance principle is chosen as the solving method. The results show that the size optimization is very effective in reducing the speed fluctuation of the mechanical arm during motion. Therefore, through the combination of topology optimization and size optimization, the mechanical arm has the characteristics of high stiffness, light weight, flexible and stable.
\end{abstract}

Keywords: Mobile Service Robot; Mechanical Arm; Particle Swarm Optimization; Optimization Performance Index; Multi-Objective Optimization.

\section{Introduction}

With the progress of science and technology and the development of robot technology, the field of robot applications continue to extend to people's daily life [1].

The working environment is no longer confined to the cold factories, workshops and other fixed places. It has entered the museum, science and technology museum, hospitals, families and entertainment. The relationship between robot and human is more and more close, and the concept of service robot is produced [2]. In foreign countries, the robot technology developed in Japan, as early as the 80s of last century, began to study the humanoid service robot. It hopes to replace the nanny, nurse and other human service robots to provide services for mankind. South Korea is promoting the industrialization of service robots, trying to let service robots into the ordinary family [3]. With the application and popularization of robot in family, hospital and other occasions, it can effectively alleviate the contradiction between supply and demand of domestic service personnel brought by aging population. The task of mobile service robot is mainly accomplished by arm cooperation. Thus, the performance of the mechanical arm has an important impact on the overall dynamic performance of the entire service robot. Most of the services of robotic robots in China are not specifically designed for the characteristics and applications of service robots. It only transplants the structure of the industrial robot arm to the service robot by experience. Service robots and industrial robots have different application fields [4-7].

Therefore, the service robot with industrial robot arm has the disadvantages of heavy weight, inflexible movement and slow response, which cannot meet the requirements of lightweight, comfortable and dexterous. The rational design of the mechanical arm structure is of great significance to improve the safety of the robotic manipulator and the dexterity of the operation. By using the basic theory of physiology, psychology, anthropometry, 
biomechanics, ergonomics, and engineering and other related disciplines, the interaction between "human-machine-environment" systems is analysed and studied. It creates a safe, comfortable, healthy and efficient working environment for the operator. Over the past decade, service robots in some developed countries and regions have been vigorous development and achieved fruitful results [8-11]. Various types of intelligent service robots have been introduced in various countries. However, the focus and direction of each country are different.

\section{Design of mechanical arm}

\subsection{Design specifications and design criteria}

The goal of this project is to design a mechanical arm for mobile robots, making it highly rigid, lightweight, flexible and stable. The design of the indicators is shown in Table 1 . It is the main basis for the subsequent design of the mechanical arm.

\begin{tabular}{|l|l|}
\hline Type & Design specifications \\
\hline Quality & Lightweight, single arm mass is not more than 15kg. \\
\hline Configuration & $\begin{array}{l}\text { The humanoid arm configuration is used, and the joint is } \\
\text { rotational form. }\end{array}$ \\
\hline Load & The maximum load that can be grabbed is $2 \mathrm{~kg}$. \\
\hline Rotating speed & The maximum angular velocity of each joint is 1.57rad/s. \\
\hline Arm length & $\begin{array}{l}\text { Compact, single arm maximum elongation is not more } \\
\text { than 650mm. }\end{array}$ \\
\hline Other & $\begin{array}{l}\text { The movement is flexible and stable, and the gripper or } \\
\text { other mechanical arm can be installed at the end. }\end{array}$ \\
\hline
\end{tabular}

The designed mechanical arm can reflect the characteristics of robot arm of mobile service robot. It should have a lighter quality, better amenity and more dexterity, instead of transplanting an industrial mechanical arm into a service robot with experience.

The mechanical arm has good mechanical properties. It avoids the difficulty of designing the control system due to the poor mechanical performance, which affects the final dynamic performance of the mobile service robot. According to the application, and combined with the relevant design theory, from the aspects of function, structure, ergonomics and aesthetics, several guidelines on the design of mobile robotic mechanical arm are proposed:

First, in function, according to the design specifications and functional requirements, the number of degrees of freedom of the robot arm is reasonably chosen. In addition, it is reasonably configured, and the optimal configuration is selected. It can simulate the human body arm to complete the basic action. The end effector has the flexibility to reach the various mission targets within the scope of the work.

Second, in the structure, the structure of the mechanical arm design should be reasonable, to avoid the structure cannot exercise and other issues. The connection of each component is simple and lightweight, which is easy loading and unloading. The simpler the structure, the better the stability.
The more compact the structure, the higher the space utilization. The more flexible the movement, the more convenient the control.

Third, in human engineering, the designed mechanical arm should have better working stability and safety, and it cannot pose a harm to people's safety. At the same time, it should have a better pleasant, to increase the user's favourability of the product, so as to obtain visual and psychological intimacy.

\subsection{Determination of the dimensions of the arm and the range of the joint rotation}

The ultimate goal of designing a service robot is to make it serve human beings. If the joint angle and shape of robot are similar to human, it has good amenity and enhances people's cordial feeling. More importantly, there is no need to design the working environment and tools for the robot alone [12].

Therefore, the size of the various parts of the arm should be as close as possible to the size of the human arm. However, the proportion of people in different countries and regions is different. It is difficult to determine a consistent size as a design standard. In view of this, in GB10000-1988 "Chinese adult human size", the size of each part of the human body is specified in detail. Table 2 is the main size of the human body arm. 
Table 2. The main size of the human body arm.

\begin{tabular}{|l|l|l|l|l|l|l|l|l|l|l|l|l|}
\hline Types & \multicolumn{4}{|l}{ Male (18-60 years old) } & \multicolumn{4}{l}{ Female (18-55 years old) } \\
\hline Percentage & 1 & 10 & 50 & 90 & 95 & 99 & 1 & 10 & 50 & 90 & 95 & 99 \\
\hline Height & 1543 & 1604 & 1678 & 1754 & 1775 & 1814 & 1543 & 1604 & 1678 & 1754 & 1775 & 1814 \\
\hline $\begin{array}{l}\text { Big arm } \\
\text { length }\end{array}$ & 279 & 294 & 313 & 333 & 338 & 349 & 279 & 294 & 313 & 333 & 338 & 349 \\
\hline $\begin{array}{l}\text { Lower } \\
\text { arm } \\
\text { length }\end{array}$ & 206 & 220 & 237 & 253 & 258 & 268 & 206 & 220 & 237 & 253 & 258 & 268 \\
\hline
\end{tabular}

Here, this is only a reference for the dimensions of the mechanical arm, and it is not the only basis for determining the size. The end users of mobile service robot are old people. Considering people hunched phenomenon in old age, for the elderly and robot interaction, the dimensions of each part in this paper are slightly smaller than those in Table 2 . The size of the robot is as follows: height is $1.7 \mathrm{~m}$, shoulder width is $0.51 \mathrm{~m}$, arm length is $0.30 \mathrm{~m}$, arm length is $0.23 \mathrm{~m}$, wrist length is $0.11 \mathrm{~m}$. In order to achieve the best performance indicators, the size of each part can be changed within a certain range.

The rotation of the joints of the human arm makes it possible to flexibly complete a variety of complex movements. In order to make the service robot arm more flexible in the process of movement, it is necessary to make each joint have a reasonable range of rotation. The torque values of each joint are shown in Table 3.

Table 3. The torque values of each joint

\begin{tabular}{|l|l|l|l|l|l|l|}
\hline Joint & Joint 1 & Joint 2 & Joint 3 & Joint 4 & Joint 5 & Joint 6 \\
\hline Corner range $^{\circ}$ ) & $-150 \sim 40$ & $-20 \sim 120$ & $-135 \sim 10$ & $-90 \sim 90$ & $-90 \sim 90$ & $-90 \sim 90$ \\
\hline
\end{tabular}

The range of motion of each joint in Table 3 is compared with the range of human arm movement. It can be seen that the angular range of each joint of the mechanical arm reaches the rotation range of the human arm. And even the range of joint rotation is greater than the angle of the arm. The mechanical arm can simulate all the complex movements of the human body arm.

\subsection{Mechanical arm structure of the material lightweight}

Reasonable selection of materials is an effective way to achieve lightweight [13]. The commonly used service robot connecting rod material is $2 \mathrm{~A} 12$ aluminium alloy.
However, the yield strength of 2A12 aluminium alloy is relatively low, which makes the structural safety factor of the designed aluminium alloy lower.

The material of the mechanical arm structure is 7075-T6 aluminium alloy with better performance in this paper. 075-T6 is reputed as the most excellent product in aluminium alloy. It has a high strength and a high light density ratio. After solution treatment, the plasticity is good, and the process ability is good. After heat treatment, the strengthening effect is obvious. It is widely used in the fields of automobile, navigation and aviation. The performance comparison between 7075-T6 aluminium alloy and 2A12 aluminium alloy material is shown in Table 4.

Table 4. Comparison of properties between 7075-T6 aluminium alloy and 2A12 aluminium alloy

\begin{tabular}{|l|l|l|l|l|}
\hline Material & Density $\rho\left(\mathrm{kg} / \mathrm{m}^{3}\right)$ & $\begin{array}{l}\text { Elastic modulus E (N } \\
\left./ \mathrm{m}^{2}\right)\end{array}$ & Poisson's ratio $\mu$ & $\begin{array}{l}\text { Yield strength } \sigma \\
\left(\mathrm{N} / \mathrm{m}^{2}\right)\end{array}$ \\
\hline 2A12 & 2830 & $7.2 \times 10^{10}$ & 0.33 & $3.45 \times 10^{8}$ \\
\hline $7075-\mathrm{T} 6$ & 2810 & $7.2 \times 10^{10}$ & 0.33 & $5.05 \times 10^{8}$ \\
\hline
\end{tabular}


As can be seen form Table 4, the yield strength of 7075-T6 aluminium alloy is obviously higher than that of 2A12 aluminium alloy, and the density is lower than that of 2A12 aluminium alloy under the same elastic modulus and Poisson's ratio. The yield strength is increased while reducing the weight of the mechanical arm.

\subsection{Topological lightweight mechanical arm structure}

Topology optimization is an effective method for obtaining high stiffness and lightweight mechanical arm. Topology optimization can reduce the quality of the robot arm, so that the total flexibility of the arm is decreased [14].

The stiffness of the mechanical arm is increased while the quality of the arm is reduced. The topology optimization process is typically to separate the design area into a sufficiently small sub-design area.

Then, according to some optimization strategy, some areas are removed from the sub-design area. The reserved area is used to express the optimal topology of the structure. Combined with the above analysis and Patran topology optimization principle, the process of topology optimization using Patran software is summarized, as shown in Figure 1.

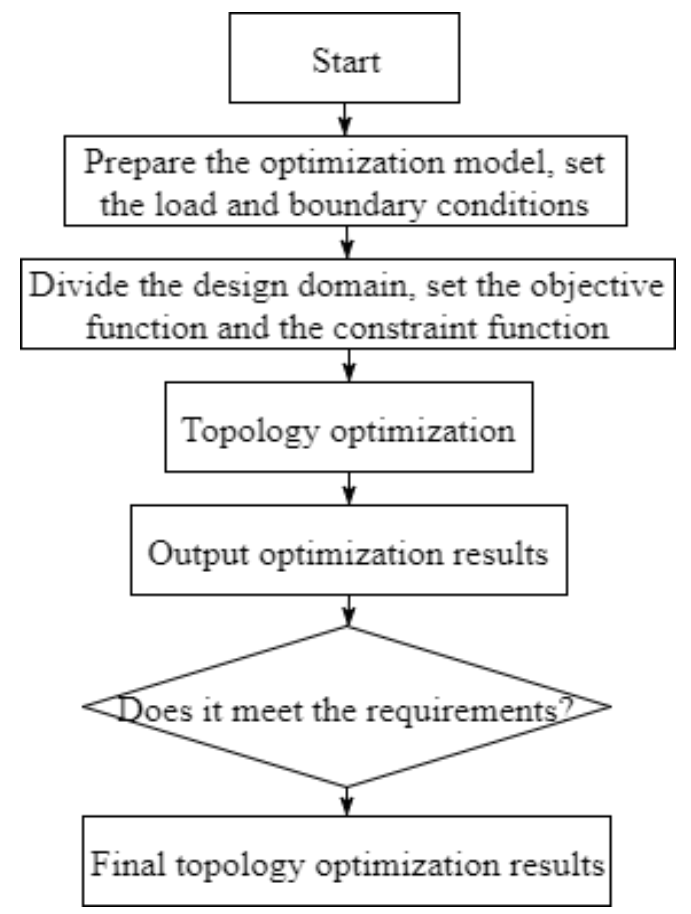

Figure 1. Flow chart of topology optimization

As can be seen from the topology optimization flow chart, it is necessary to determine the topological optimization design area and non- design area according to the actual structure before topology optimization. The lower arm is made up of two separate parts.

The design area is relatively small. The effect of topology optimization is not obvious. Therefore, only the big arm is optimized by topology. The connection between the big arm and the drive module is defined as a non-design area. The rest is defined as the design area.

Patran software has a very powerful pre-and post-processing capabilities. The 3D arm model of Solidworks software is imported into Patran software [15]. The big arm is cut according to the design area and non-design area. 7075-T6 aluminium alloys are used in both design and nondesign areas. After the topology optimization, the iterative process of the objective function can be obtained according to the information provided by the result file, as shown in Figure 2.

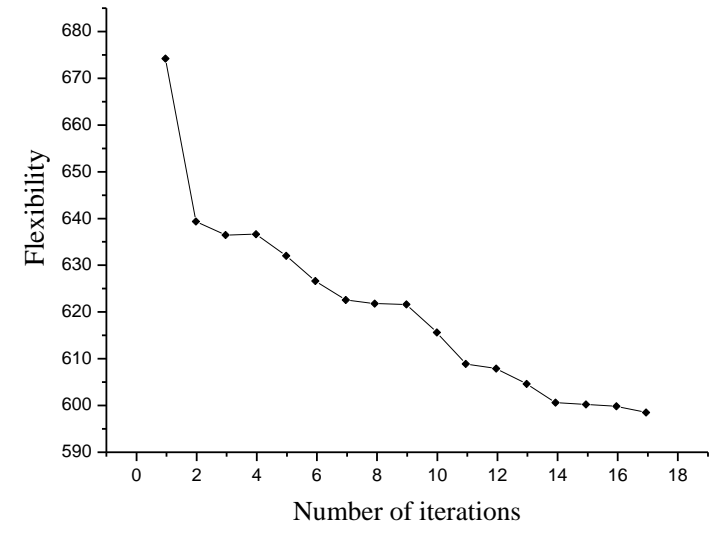

Figure 2. Iterative process of topology optimization for big arms

It can be seen that the flexibility of the bog arm after optimization is reduced, that is, the static stiffness increases. Topology optimization belongs to the category of conceptual design, and the results cannot be directly applied to the actual mechanical arm. It also needs to consider the feasibility of manufacturing, processing technology, ease of production and other factors to redesign the topology optimization structure. The following structural re-design ideas are provided:

First, the pseudo-density value of the larger units is mainly concentrated in the design area of the two sides, which shows that both sides bear a greater force. Therefore, the material should be distributed mainly in both sides of the design area.

Second, in the process of adjusting the topology-optimized material volume threshold, the unit in the middle of the design side of the two walls disappears first. Thus, on the sidewall, the intermediate material can also be properly removed. 
Thirdly, in order to enhance the strength of the boom, there is a stiffener in the design area. At the same time, a special reinforcement is applied to the design area and the non-design area, in order to avoid stress concentration at the structural transition.

\section{Experiment}

\subsection{Quality constraint condition}

One of the objectives of this project is to realize the lightweight of the robot arm. The speed fluctuation of the mechanical arm cannot be reduced by increasing the weight of the mechanical arm. The work space cannot be increased by increasing the quality of the mechanical arm. That is to say, the size of the big arm and the lower arm after the size optimization cannot be greater than the quality before optimization. In the previous chapter, the lightweight of the big arm and the whole mechanical arm are realized from two aspects of topological lightweight and material lightweight. In this case, it is treated only as a constraint.

$$
m_{1} \leq m_{10}, m_{2} \leq m_{20}
$$

In formula (1), $\mathrm{m}_{10}$ is the mass before the optimization of the big arm, and $m_{1}$ is the mass after the optimization of the big arm. $\mathrm{m}_{20}$ is the mass before the optimization of the lower arm, and $m_{2}$ is the mass after the optimization of the lower arm.

Using Solidworks software, the mass of the big arm before optimization is $m_{10}=1.777 \mathrm{~kg}$, and the mass of the lower arm before optimization is $\mathrm{m}_{20}=1.777 \mathrm{~kg}$. In order to optimize the convenience, the constraints are transformed into the form related to the design variables. Because the actual shape of the robot arm is complex, it is necessary to simplify the structure in the process of conversion. According to the geometrical characteristics of the big arm and lower arm, the big arm is simplified as a hollow beam with constant cross section, and the lower arm is simplified as a hollow cylinder with constant cross section. Before and after simplification, the mass, centroid position and inertial tensor of the arms and sizes are consistent with the original parameters.

The simplified big arm diagram of a mobile service robot is shown in Figure 3. The simplified lower arm diagram of a mobile service robot is shown in Figure 4.

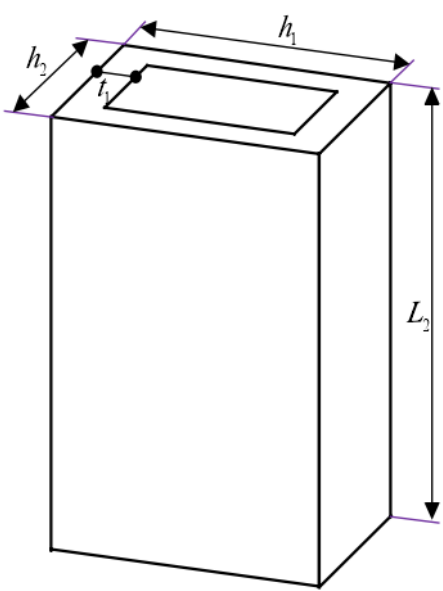

Figure 3. The simplified big arm diagram of a mobile service robot

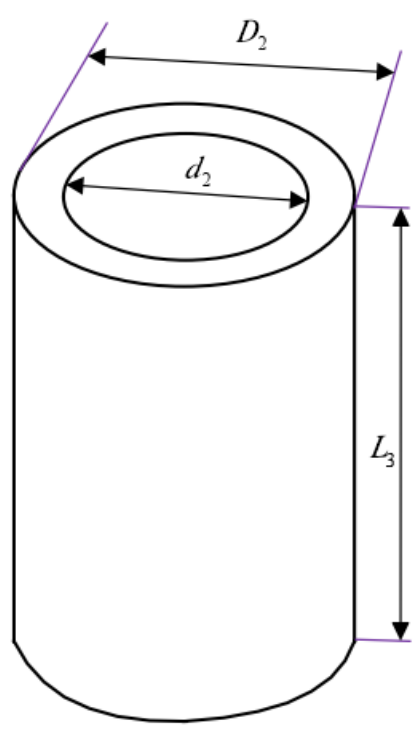

Figure 4. The simplified lower arm diagram of a mobile service robot

\subsection{Simulation verification and result analysis}

After the trajectory planning is completed, consider the angle range of each joint, the inverse kinematics is used to find the variation curve of the joint angular displacement of the mechanical arm. It is introduced into the Adams simulation software as a data point and is fitted into a spline curve as the driving of each joint. The Adams simulation is carried out. The angular velocity curve of each joint is shown in Figure 5. The angular velocity of each joint changes is as shown in Table 5. 


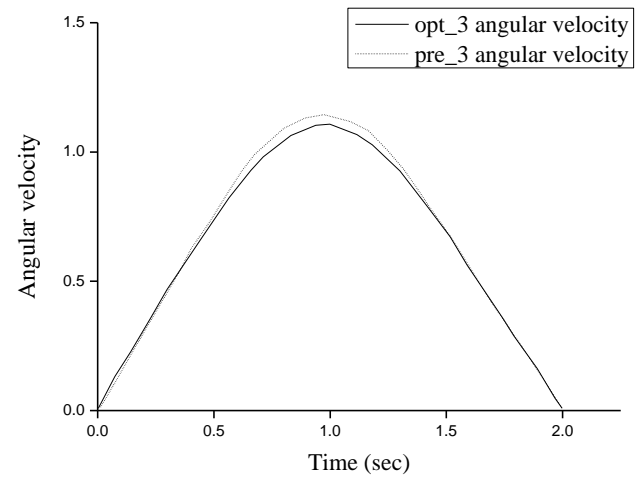

(a) The angular velocity curve of joint 3

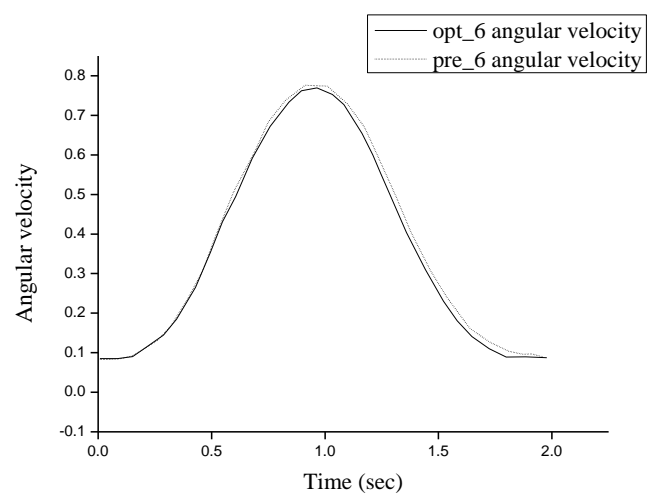

(b) The angular velocity curve of joint 6 Figure 5. The angular velocity curve of each joint

Table 5. The angular velocity of each joint changes

\begin{tabular}{|l|l|l|l|l|l|l|}
\hline Angular velocity index & Joint 1 & Joint 2 & Joint 3 & Joint 4 & Joint 5 & Joint 6 \\
\hline $\begin{array}{l}\text { Mean value before } \\
\text { optimization }\end{array}$ & 0.7162 & 0.1473 & 0.6601 & 0.5233 & 0.4738 & 0.3501 \\
\hline $\begin{array}{l}\text { Mean value after } \\
\text { optimization }\end{array}$ & 0.6790 & 0.1449 & 0.6484 & 0.4933 & 0.4511 & 0.3257 \\
\hline $\begin{array}{l}\text { Mean difference before and } \\
\text { after optimization }\end{array}$ & $\downarrow 0.0372$ & $\downarrow 0.0024$ & $\downarrow 0.0117$ & $\downarrow 0.0300$ & $\downarrow 0.0227$ & $\downarrow 0.0244$ \\
\hline Percentage change & $5.1941 \%$ & $1.6293 \%$ & $1.7725 \%$ & $5.7328 \%$ & $4.7911 \%$ & $6.9694 \%$ \\
\hline $\begin{array}{l}\text { Root mean square before } \\
\text { optimization }\end{array}$ & 0.7865 & 0.1664 & 0.7467 & 0.6392 & 0.5611 & 0.4384 \\
\hline $\begin{array}{l}\text { Root mean square after } \\
\text { optimization }\end{array}$ & 0.7661 & 0.1639 & 0.7460 & 0.6161 & 0.5472 & 0.4139 \\
\hline $\begin{array}{l}\text { Root mean square difference } \\
\text { before and after optimization }\end{array}$ & $\downarrow 0.0204$ & $\downarrow 0.0025$ & $\downarrow 0.0007$ & $\downarrow 0.0231$ & $\downarrow 0.0139$ & $\downarrow 0.0245$ \\
\hline Percentage change & $2.5938 \%$ & $1.5024 \%$ & $0.0937 \%$ & $3.6139 \%$ & $2.4773 \%$ & $5.5885 \%$ \\
\hline
\end{tabular}

In Figure 5, the solid line represents the angular velocity curve of each joint of the optimized arm, and the dotted line represents the angular velocity curve of each joint of the optimized front arm. It can be seen from the figure that the angular velocity amplitude of each joint before optimization is different in different degrees.

As can be seen from Table 5 , the angular velocity of joint 3 decreases by a maximum of $3.0255 \%$. The amplitude of the angular velocity of joint 6 is the smallest, which is $0.9335 \%$. The angle amplitude of the rest joints decreased by $1 \% \sim 3 \%$. The average angular velocity and the mean square root of each joint before and after optimization are reduced to varying degrees. The root means square value of the angular velocity of the joint 6 is the largest. After optimization, it decreased by $5.5885 \%$. The root means square value of the angular velocity of the joint 3 before and after optimization is the smallest.

After optimization, it decreased by $0.0937 \%$. The range of the angular velocity before and after the optimization of the rest joints is between $1 \% \sim 3 \%$. In general, the speed fluctuation of the manipulator is reduced by optimization, and the optimization effect is obvious.

\section{Conclusion}

The main goal of this paper is to design a mechanical arm for mobile service robot. It has the characteristics of high rigidity, light weight, flexible and smooth movement. Based on the application situation and characteristics of mobile service robot, the design criteria, optimal configuration, topology optimization, finite element analysis, size optimization and optimization algorithm are studied. The structural design criteria of mobile service robot arm in accordance with human engineering requirements are proposed. The optimal configuration of the manipulator is obtained, which makes it have the flexibility similar to human. The multi-objective particle swarm optimization algorithm is perfected to improve the accuracy of the algorithm. The improved algorithm is used to solve the size optimization model. By comparing the before and after velocity fluctuation index values and the simulation experiments, the feasibility and effectiveness of the size optimization suppression velocity fluctuation are verified. 
Through the combination of topology optimization and performance index size optimization, the designed manipulator has the characteristics of high stiffness, light weight, flexibility and stability.

\section{References}

[1] Du, H., Zhang, Y., Cai, W., Chen, H., \& Lin, X. (2017). The trajectory optimization of mobile crossbeam in composite hydraulic press based on modified multiisland genetic algorithm. Journal of the Chinese Institute of Engineers, 40(3), 219-227.

[2] Zavala, G. R., Nebro, A. J., Luna, F., \& Coello, C. A. C. (2014). A survey of multi-objective metaheuristics applied to structural optimization. Structural and Multidisciplinary Optimization, 49(4), 537-558.

[3] Lim, Z. Y., Ponnambalam, S. G., \& Izui, K. (2016). Nature inspired algorithms to optimize robot work cell layouts. Applied Soft Computing, 49, 570-589.

[4] Li, Y., Yao, X., \& Zhou, J. (2016). Multi-objective optimization of cloud manufacturing service composition with cloud-entropy enhanced genetic algorithm. Strojniški vestnik-Journal of Mechanical Engineering, 62(10), 577-590.

[5] Burget, F., Fiederer, L. D. J., Kuhner, D., Völker, M., Aldinger, J., Schirrmeister, R. T., ... \& Burgard, W. (2017). Acting Thoughts: Towards a Mobile Robotic Service Assistant for Users with Limited Communication Skills. arXiv preprint arXiv:1707.06633.

[6] Cui, L., Tan, T., Do, K., \& Teunissen, P. (2015). Challenges and solutions for autonomous robotic mobile manipulation for outdoor sample collection. Journal of Electrical and Electronic Engineering, 3(5), 156-164.

[7] Wise, M., Ferguson, M., King, D., Diehr, E., \& Dymesich, D. (2016). Fetch and freight: Standard platforms for service robot applications. In Workshop on Autonomous Mobile Service Robots.
[8] Chung, H. Y., Hou, C. C., \& Chen, Y. S. (2015). Indoor intelligent mobile robot localization using fuzzy compensation and Kalman filter to fuse the data of gyroscope and magnetometer. IEEE Transactions on Industrial Electronics, 62(10), 6436-6447.

[9] Xidias, E. K., Azariadis, P. N., \& Aspragathos, N. A. (2016). Mission Design of Mobile Manipulators in Cluttered Environments for Service Applications. International Journal of Robotics Applications and Technologies (IJRAT), 4(1), 1-18.

[10] Rodić, A., Jovanović, M., Stevanović, I., Karan, B., \& Potkonjak, V. (2015). Building technology platform aimed to develop service robot with embedded personality and enhanced communication with social environment. Digital communications and networks, 1(2), 112-124.

[11] Carbone, G., Tedeschi, F., Gallozzi, A., \& Cigola, M. (2015). A robotic mobile platform for service tasks in cultural heritage. International Journal of Advanced Robotic Systems, 12(7), 88.

[12] Bogue, R. (2015). Sensors for robotic perception. Part one: human interaction and intentions. Industrial Robot: An International Journal, 42(5), 386-391.

[13] Rahman, M. H., Saad, M., Kenné, J. P., \& Archambault, P. S. (2013). Control of an exoskeleton robot arm with sliding mode exponential reaching law. International Journal of Control, Automation and Systems, 11(1), 92-104.

[14] Kristoffersson, A., Coradeschi, S., \& Loutfi, A. (2013). A review of mobile robotic telepresence. Advances in Human-Computer Interaction, 2013, 3.

[15] Kumari, M. P., \& Paul, R. K. (2017). Optimization of Small Scale Concentrated Solar Power Plant with Revolving Mechanical Arm. IJOSCIENCE (INTERNATIONAL JOURNAL ONLINE OF SCIENCE) Impact Factor: 3.462, 2(8), 1-8. 